\title{
Remarks on the Global Markov Property
}

\author{
Sheldon Goldstein*
}

Department of Mathematics, Rutgers University, New Brunswick, NJ 08903, USA

\begin{abstract}
The global Markov property is established for the + state and the state of attractive lattice systems (e.g., the ferromagnetic Ising model and most other systems for which the FKG inequalities are satisfied) and of the (continuum) Widom Rowlinson model.
\end{abstract}

\section{Introduction}

Consider the Gibbs states for a nearest neighbor interaction $\Phi$ on the lattice $\Gamma=\mathbb{Z}^{d}$ with finite state space $S$. These are the probability measures $\mu$ on $\Omega=S^{\Gamma}$ whose conditional probabilities satisfy the DLR equations $[1,2]$ :

$$
\mu\left\{\sigma_{\Lambda}=y \mid x_{\Gamma-\Lambda}\right\}=\frac{e^{-h_{\Lambda}^{\Phi}(y, x)}}{\sum_{y^{\prime} \in \Omega_{\Lambda}} e^{-h_{\Lambda}^{\Phi}\left(\left(^{\prime}, x\right)\right.}} .
$$

Here $\Lambda$ is a finite subset of $\Gamma, \Omega_{\Lambda}=S^{\Lambda}$ is the set of configurations on $\Lambda, y \in \Omega_{\Lambda}, x \in \Omega$, $x_{\Gamma-\Lambda}$ is the configuration in $\Omega_{\Gamma-\Lambda}$ obtained by restricting $x$ to $\Gamma-\Lambda, \sigma_{\Lambda}(x)=x_{\Lambda}$, and $h_{\Lambda}^{\Phi}(y, x)$ is the energy in $\Lambda$ produced by the interaction $(\Phi)$ of the spins in $\Lambda$ (described by $y$ ) with themselves and with the spins outside of $\Lambda$ (described by $\left.x_{\Gamma-\Lambda}\right)$. In particular the state $\mu$ satisfies the local Markov property: For any bounded $f \in \mathscr{F}_{F}$,

$$
E\left(f \mid \mathscr{F}_{\Gamma-F}\right)=E\left(f \mid \mathscr{F}_{\partial F}\right),
$$

where $F=\Lambda$ is a finite subset, $\partial F$ s the set of sites in $\Gamma-F$ which are nearest neighbors to at least one site in $F, \mathscr{F}_{D}$ is the sub- $\sigma$-algebra generated by $\sigma_{D}, D \subset \Gamma$, $E\left(\cdot \mid \mathscr{F}_{D}\right)$ denotes the conditional expectation given $\mathscr{F}_{D}$, and $f \in \mathscr{F}_{F}$ means $f$ is bounded and $\mathscr{F}_{F}$ measurable. We write $\mathscr{F}_{\text {for }} \mathscr{F}_{\Gamma}$.

Markov chains by definition satisfy (1.2) $(d=1)$ for $F=\{n, n+1, n+2, \ldots\}$ and, in fact, satisfy (1.2) for any $F$ whatsoever. A state $\mu$ satisfying (1.2) for any (not

* Supported in part by NSF Grant No. PHY 78-03816 
necessarily bounded) set $F \subset \Gamma$ is said to satisfy the global Markov property. Albeverio and Høegh-Krohn [3] have shown that if $\Phi$ satisfies Dobrushin's uniqueness condition then the Gibbs state satisfies the global Markov property. Dobrushin's condition, which of course cannot be satisfied if there is a phase transition, is very strong and may also fail to be satisfied even where there is a unique Gibbs state. On the other hand, it appears reasonable to conjecture that every extremal Gibbs state satisfies the global Markov property. In Sect. 2 we give an example of a Gibbs state for the Ising model (with $d=3$ ) which does not satisfy the global Markov property. In Sect. 3 we indicate why we believe it likely that every extremal Gibbs state satisfies the global Markov property. In Sect. 4 we show that the + state and the - state for attractive systems satisfy the global Markov property, and in Sect. 5 we extend the results to the Widom Rowlinson Model - a continuum system which exhibits a phase transition.

The results of Sect. 4 are similar to those of Follmer [4], of which we became aware after our results were obtained.

A bit more notation: We will denote by $\mu_{\Lambda}(\cdot \mid x)$ the measure on $\Omega_{\Lambda}$ described by the RHS of (1.1). $\Lambda_{n}$ will always denote an increasing sequence of cubes centered at the origin with $\bigcup_{n} \Lambda_{n}=\Gamma$.

\section{An Example}

Let $d=3, S=\{ \pm 1\}, \Phi$ a n.n. ferromagnetic Ising interaction :

$$
h_{\Lambda}^{\Phi}(y, x)=-\frac{1}{k T}\left(\sum_{\substack{i<j \\ i, j \in \Lambda}} y_{i} y_{j}+\sum_{\substack{i \in \Lambda \\ j \in \partial \Lambda}} y_{i} x_{j}\right) .
$$

Let the Gibbs state $\mu_{-}^{+}$be the limit $n \rightarrow \infty$ of the measures $\mu_{\Lambda_{n}}(\cdot \mid \bar{x})$, where for $i=\left(i_{1}, i_{2}, i_{3}\right) \in \mathbb{Z}^{3}, \bar{x}_{i}=+1$ for $i_{3} \geqq 0$ and $\bar{x}_{i}=-1$ for $i_{3}<0$. Dobrushin [5] has shown that $\mu_{-}^{+}$is not translation invariant, looking more and more like the + state (which arises from the boundary condition $x_{i}=+1$ ) as $i_{3} \rightarrow \infty$ and more and more like the - state as $i_{3} \rightarrow-\infty$. Let $\mu_{+}^{-}$be the Gibbs states obtained from $\mu_{-}^{+}$by the reflection $\left(i_{1}, i_{2}, i_{3}\right) \rightarrow\left(i_{1}, i_{2},-i_{3}\right)$. Note that since this reflection is the identity on $\left\{i_{3}=0\right\}, \mu_{-}^{+}$agrees with $\mu_{+}^{-}$on $\mathscr{F}_{\left\{i_{3}=0\right\}}$. Let $\mu=\frac{1}{2}\left(\mu_{-}^{+}+\mu_{+}^{-}\right)$. Then $\mu$ is a Gibbs state failing to satisfy the global Markov property. In fact, (1.2) fails for $F=\left\{i_{3}>0\right\}$, since $\mu_{-}^{+}$and $\mu_{+}^{-}$, which agree on $\mathscr{F}_{\partial F}$, are mutually singular on $\mathscr{F}_{T-F}$, so that specifying $x_{\Gamma-F}$ determines whether $x \in \Omega$ is a configuration of the $\mu_{-}^{+}$phase or of the $\mu_{+}^{-}$phase. Note that $\mu$ is not extremal.

\section{A Sufficient Condition}

Suppose $\mu$ is a Gibbs state, $x^{0} \in \Omega, F \subset \Gamma$. We denote by $\mu_{\Lambda, F}\left(\cdot \mid x^{0}\right)$ the probability measure on $\Omega_{\Lambda}$ which on $\mathscr{F}_{\Lambda-F}$ agrees with $\mu$ and for which the conditional probability given $\mathscr{F}_{\Lambda-F}$ is given by $\mu_{\Lambda \cap F}\left(\cdot \mid x_{\Lambda-F} x_{\Gamma-(\Lambda-F)}^{0}\right)$, where $x_{\Lambda-F} x_{\Gamma-(\Lambda-F)}^{0}$ is the configuration which agrees with $x$ on $\Lambda-F$ and with $x^{0}$ elsewhere. In other words, for $f \in \mathscr{F}_{\Lambda \cap F}, g \in \mathscr{F}_{\Lambda-F}$,

$$
\mu_{\Lambda, F}\left(g f \mid x^{0}\right)=\int \mu(d x) g(x) \mu_{\Lambda \cap F}\left(f \mid x_{\Lambda-F} x_{\Gamma-(\Lambda-F)}^{0}\right) .
$$


We will say that the Gibbs state $\mu$ satisfies condition (C) if there exists $x^{0} \in \Omega$ such that

(i) $\mu=\lim _{n \rightarrow \infty} \mu_{\Lambda_{n}}\left(\cdot \mid x^{0}\right)$

and

(ii) for every $F \subset \Gamma, \quad \lim _{n \rightarrow \infty} \mu_{\Lambda_{n}, F}\left(\cdot \mid x^{0}\right)=\mu$.

Proposition 3.1. Suppose the Gibbs state $\mu$ satisfies condition (C). Then $\mu$ satisfies the global Markov property.

Proof. Fix $F \subset \Gamma$. Showing that

$$
E\left(f \mid \mathscr{F}_{\Gamma-F}\right) \in \mathscr{F}_{\partial F}
$$

for every function $f \in \bigcup_{n} \mathscr{F}_{F \cap \Lambda_{n}}$, i.e., that for every function $f \in \mathscr{F}_{F \cap \Lambda_{n}}$, there exists a function $f_{\infty} \in \mathscr{F}_{\partial F}$ such that

$$
\mu(g f)=\mu\left(g f_{\infty}\right)
$$

for every function $g \in \bigcup_{m} \mathscr{F}_{\Lambda_{m}-F}$, will suffice to establish (1.2).

Fix an $x^{0}$ such that condition (C) is satisfied. Let $f \in \mathscr{F}_{F \cap \Lambda_{m}}$ and let

$$
f_{n}(x)=\mu_{\Lambda_{n} \cap F}\left(f \mid x_{\Lambda-F} x_{\Gamma-(\Lambda-F)}^{0}\right)
$$

for $n \geqq m$. It follows from (3.1) and (3.3) that (3.5) will be satisfied by

$$
f_{\infty}=\lim _{n \rightarrow \infty} f_{n}(x),
$$

and $f_{\infty} \in \mathscr{F}_{\partial F}$ since $f_{n} \in \mathscr{F}_{(\partial F) \cap \Lambda_{n}}$, provided this limit exists. In many models, e.g., those described in Sect. 4, the existence of the limit (3.6) may be easily established. However, this is not necessary, since (3.1) and (3.3) imply in any case that

$$
\lim _{n \rightarrow \infty} \mu\left(g f_{n}\right)=\mu(g f)=\mu\left(g E\left(f \mid \mathscr{F}_{\Gamma-F}\right)\right)
$$

for all $g \in \bigcup_{m} \mathscr{F}_{\Lambda_{m}-F}$, i.e., that $f_{n}$ converges weakly to $E\left(f \mid \mathscr{F}_{\Gamma-F}\right)$ in $L^{2}\left(\mu, \mathscr{F}_{\Gamma-F}\right)$. Since subspaces are weakly closed, (3.4) follows.

Note that

$$
\mu_{\Lambda}\left(g f \mid x^{0}\right)=\int \mu_{\Lambda}\left(d x_{\Lambda}\right) g(x) \mu_{\Lambda \cap F}\left(f \mid x_{\Lambda-F} x_{\Gamma-(\Lambda-F)}^{0}\right)
$$

for $f \in \mathscr{F}_{\Lambda \cap F}$ and $g \in \mathscr{F}_{\Lambda-F}$. Comparing (3.8) with (3.1), we see that $\mu_{\Lambda, F}\left(\cdot \mid x^{0}\right)$ differs from $\mu_{\Lambda}\left(\cdot \mid x^{0}\right)$ only in that part of the system, $\Omega_{\Lambda-F}$, is distributed according to $\mu$ rather than $\mu\left(\cdot \mid x^{0}\right)$. Thus $\mu_{\Lambda, F}\left(\cdot \mid x^{0}\right)$ should be closer to $\mu$ than $\mu_{\Lambda}\left(\cdot \mid x^{0}\right)$, so that if (3.2) holds so should (3.3). We thus conjecture that every Gibbs state $\mu$ of the form (3.2) for some fixed boundary condition $x^{0} \in \Omega$, and in particular every extremal Gibbs state, satisfied the global Markov property. 


\section{Attractive Interactions}

Let $S$ be partially ordered with minimal element - and maximal element + . The partial order on $S$ induces a partial order on $\Omega$ (or on $\Omega_{\Lambda}$ ):

$x \leqq y \Leftrightarrow x_{i} \leqq y_{i}$ all $i$.

A function $f$ on $\Omega$ (or on $\Omega_{A}$ ) is called increasing if

$$
x \leqq y \Rightarrow f(x) \leqq f(y) .
$$

We denote by $\mathscr{F}_{D}^{\uparrow}$ the increasing functions $\in \mathscr{F}_{D}$. Let $\mu$ and $v$ be probability measures on $\Omega$ (or on $\Omega_{\Lambda}$ ). Then we define a relation " $\leqq$ " on probability measures by

$$
\mu \leqq v \Leftrightarrow \mu(f) \leqq v(f) \text { for all } f \in \mathscr{F}^{\uparrow}\left(\mathscr{F}_{\Lambda}^{\uparrow}\right)
$$

$\left[" \leqq "\right.$ is a partial order on probability measures on $\Omega\left(\Omega_{\Lambda}\right)$ since two measures on $\Omega\left(\Omega_{\Lambda}\right)$ which agree on $\mathscr{F}^{\dagger}\left(\mathscr{F}_{\Lambda}^{\uparrow}\right)$ coincide.]

An interaction $\Phi$ is called attractive if

$$
x \leqq y \Rightarrow \mu_{\Lambda}(\cdot \mid x) \leqq \mu_{\Lambda}(\cdot \mid y)
$$

for all bounded $\Lambda \subset \Gamma$.

For an attractive interaction $\mu_{\Lambda}(\cdot \mid+)^{\star} \leqq \mu_{\Lambda^{\prime}}(\cdot \mid+)$ when $\Lambda^{\prime} \subset \Lambda$ and hence the + state

$$
\mu^{+}=\lim _{n \rightarrow \infty} \mu_{\Lambda_{n}}(\cdot \mid+),
$$

and similarly the - state $\mu^{-}$, is well defined. (We use + to denote the configuration $x_{i}=+$, all $i$.)

Theorem 4.1. The + state and the - state for a system with n.n. attractive interaction satisfy the global Markov property.

Proof. We need only show that (3.3) is satisfied by $\mu=\mu^{+}$and $x^{0}=+$. But this follows immediately by passing to the limit $n \rightarrow \infty$ in

$$
\mu_{\Lambda_{n}}(\cdot \mid+) \geqq \mu_{\Lambda_{n}, F}(\cdot \mid+) \geqq \mu \quad \text { on } \Omega_{\Lambda_{n}} .
$$

The first inequality follows, using (3.1), from the facts that $\mu_{\Lambda_{n}}(\cdot \mid+) \geqq \mu^{+}$on $\mathscr{F}_{\Lambda_{n}}$ and that for $f \in \mathscr{F}_{\Lambda_{n}}$, the function

$$
x \rightarrow \int \mu_{\Lambda_{n} \cap F}\left(d y_{A_{n} \cap F} \mid x_{A_{n}-F}{ }_{\Gamma-\left(\Lambda_{n}-F\right)}\right) f\left(y_{A_{n} \cap F} x_{\Lambda_{n}-F}\right) \in \mathscr{F}_{\Lambda_{n}}^{\uparrow} .
$$

Since

$$
\mu_{\Lambda_{n} \cap F}(\cdot \mid x) \leqq \mu_{\Lambda_{n} \cap F}\left(\cdot \mid x_{\Lambda_{n}-F}+{ }_{\Gamma-\left(\Lambda_{n}-F\right)}\right)
$$

and

$$
\mu(g f)=\int \mu(d x) g(x) \mu_{\Lambda_{n} \cap F}(f \mid x)
$$

for $g \in \mathscr{F}_{\Gamma-\left(A_{n} \cap F\right)}$ and $f \in \mathscr{F}_{\Lambda_{n} \cap F}$, comparison with (3.1) yields the second inequality.

$\star \quad$ Regarded as a measure on $\Omega_{\Lambda^{\prime}}$ 
Examples. The best known examples of attractive interactions are those for which the FKG inequalities [6] are satisfied. These inequalities say that

$$
\langle f g\rangle \geqq\langle f\rangle\langle g\rangle
$$

where $\langle\cdot\rangle=\mu_{\Lambda}(\cdot \mid x)$ and $f$ and $g$ are $\in \mathscr{F}_{\Lambda}^{\uparrow}$. Note that for $g \geqq 0,\langle g\rangle \neq 0,(4.5)$ says that

$$
\langle\cdot\rangle \leqq\langle\cdot\rangle_{g},
$$

where

$$
\langle\cdot\rangle_{g}=\frac{\langle\cdot g\rangle}{\langle g\rangle} .
$$

It therefore follows from (1.1) that if the FKG inequalities are satisfied, and if $x \leqq x^{\prime}$ implies that $h_{\Lambda}^{\Phi}(y, x)-h_{\Lambda}^{\Phi}\left(y, x^{\prime}\right)$ is increasing in $y$, then $\Phi$ is attractive; this is because if $\mu_{\Lambda}(\cdot \mid x)=\langle\cdot\rangle$ then $\mu_{\Lambda}\left(\cdot \mid x^{\prime}\right)=\langle\rangle_{g}$, where $g=\exp \left(h_{\Lambda}^{\Phi}(y, x)-h_{\Lambda}^{\Phi}\left(y, x^{\prime}\right)\right)$. Moreover if the "FKG condition" $[6], \mu(a \vee b) \mu(a \wedge b) \geqq \mu(a) \mu(b)$, is satisfied by all the $\mu_{\Lambda}(\cdot \mid x)$ 's, then $\Phi$ is attractive since we then have (I) $x \leqq x^{\prime} \Rightarrow h_{\Lambda}^{\Phi}(y, x)-h_{\Lambda}^{\Phi}\left(y, x^{\prime}\right)$ is increasing in $y$. In fact, the satisfaction of (I) for all $\Lambda$ is equivalent to the "FKG condition" and hence implies the attractiveness of $\Phi$. In particular, if $S \subset \mathbb{R}$, and if

$$
\frac{\partial}{\partial x_{i}}\left(-h_{\Lambda}^{\Phi}(y, x)\right)
$$

is increasing in $y, \Phi$ is attractive. We are assuming here that $h_{\Lambda}^{\Phi}(y, x)$ makes sense (and is $C^{1}$ ) for all values of the $x_{i}$, not just those in $S$.

Suppose $S \subset \mathbb{R}$, and $h_{\Lambda}^{\Phi}(y, x)$ is defined and is $C^{2}$ as a function of $x_{i}, y_{j} \in \mathbb{R}$, all $i, j$. It follows from the above that $\Phi$ is attractive if

$(*) \frac{\partial^{2}\left(-h_{\Lambda}^{\Phi}(y, x)\right)}{\partial y_{j} \partial x_{i}} \geqq 0$.

(See also [7].)

Example 4.1. Suppose $S \subset[0, \infty)$, and let

$$
h_{A}^{\Phi}(y, x)=-\sum_{\substack{A \subset \Gamma \\ A \cap A \neq \emptyset}} J_{A} \varrho_{A},
$$

where the sum is over finite subsets $A$ of $\Gamma$,

$$
\varrho_{A}(y, x)=\prod_{i \in A \cap A} y_{i} \prod_{j \in A \cap(T-\Lambda)} x_{j},
$$

and $J_{A} \geqq 0$ for sets $A$ containing two or more points. Since $(*)$ is satisfied $\Phi$ is attractive.

Example 4.2. Suppose $S=\{-p,-p+2, \ldots, p-2, p\}$ for some positive integer $p$. It is shown in [8] that the FKG inequalities are satisfied if

$$
\begin{aligned}
h_{\Lambda}^{\Phi}(y, x)= & -\frac{1}{2} \sum_{\substack{i \neq j \\
i, j \in \Lambda}}\left(J(i, j) y_{i} y_{j}+\gamma(i, j) y_{i}^{2} y_{j}^{2}\right) \\
& -\sum_{i \in \Lambda} h(i) y_{i}-\sum_{i \in \Lambda} u(i) y_{i}^{2} \\
& -\sum_{\substack{i \in \Lambda \\
j \in I}}\left(J(i, j) y_{i} x_{j}+\gamma(i, j) y_{i}^{2} x_{j}^{2}\right),
\end{aligned}
$$


where

$$
J(i, j) \geqq 4(p-1)^{2}|\gamma(i, j)|
$$

for all $i, j \in \Lambda$. Since

$$
\begin{aligned}
\frac{\partial^{2}}{\partial y_{j} \partial x_{i}}\left(-h_{\Lambda}^{\Phi}(y, x)\right) & =J(i, j)+4 \gamma(i, j) y_{i} x_{i} \\
& \geqq J(i, j)-4|\gamma(i, j)| p^{2}
\end{aligned}
$$

we see that $\Phi$ is attractive provided $J(i, j) \geqq 4|\gamma(i, j)| p^{2}$ for all $i, j \in \Gamma$. A more detailed calculation shows that $\Phi$ is attractive as long as (4.7) is satisfied for all $i, j \in \Gamma$ : Suppose $x^{\prime}$ differs from $x$ only in that $x_{i}^{\prime}=x_{i}+2$. Note that $-p \leqq x_{i} \leqq p-2$. Then $h_{\Lambda}^{\Phi}(y, x)-h_{\Lambda}^{\Phi}\left(y, x^{\prime}\right)$ is a sum of terms of the form

$$
2 f\left(y_{j}, x_{i}\right)=2\left(J(i, j) y_{j}+2 \gamma(i, j) y_{j}^{2}\left(x_{i}+1\right)\right) .
$$

Since $f\left(y_{j}, x_{i}\right)$ is quadratic in $y_{j}$ with

$$
\begin{aligned}
\frac{\partial f}{\partial y_{j}} & =J(i, j)+4 \gamma(i, j) y_{j}\left(x_{i}+1\right) \\
& \geqq J(i, j)-4|\gamma(i, j)|(p-1)^{2} \geqq 0,
\end{aligned}
$$

for $-p+1 \leqq y_{j} \leqq p-1$, it follows that $f\left(y_{j}, x_{i}\right)$ is an increasing function of $y_{j} \in S$. (The vertex of the parabola occurs for $\left|y_{j}\right| \geqq p-1$.)

The above result also follows from the fact that the model $(4.6,7)$ satisfies the "FKG condition", as shown in [8]. The preceding calculation also provides a simple proof of this fact.

\section{The Widom Rowlinson Model}

The proof in Sects. 3 and 4 of the global Markov property depended upon the attractiveness of $\Phi$ and the existence of a maximal measure $\mu_{\Lambda}(\cdot \mid+)$, but not essentially on the lattice structure $\Gamma=\mathbb{Z}^{d}$. We here prove the global Markov property for the $+(A)$ state and the $-(B)$ state of the Widom Rowlinson model, a continuum model for a system of two kinds of particles, $A$ and $B$, which (for $d \geqq 2$ ) has been shown [9] to have a phase transition at low temperatures.

Our setup is now the following: $\Gamma=\mathbb{R}^{d}, S=\{-2,0,2\}$, and $\Omega=$ the set of functions $x: \Gamma \rightarrow S$ which are locally finite in this sense: For every set $D \subset \Gamma$ let $N_{\alpha, D}(x), \alpha= \pm 2$, be the number of points $\mathbf{r} \in D$ such that $x(\mathbf{r})=\alpha$. Let $N_{D}=N_{2, D}$ $+N_{-2, D} \cdot x$ is locally finite if $N_{D}(x)$ is finite for all bounded sets $D \subset \Gamma$. We interpret $x \in \Omega$ as a configuration of $A$ and $B$ particles: $x(\mathbf{r})=2,-2$, or 0 according to whether there is an $A$ particle, $B$ particle, or no particle at $\mathbf{r}$. We denote by $\hat{\Omega}$ the set of all functions $x: \Gamma \rightarrow S$.

Let $D$ be a Borel subset of $\Gamma$. We denote by $x_{D}$ the restriction to $D$ of $x \in \hat{\Omega}$, and by $\mathscr{F}_{D}$ the smallest $\sigma$-algebra (on $\Omega$ ) with respect to which the functions $N_{\alpha, K}$, $\alpha= \pm 2, K$ a Borel subset of $D$, are all measurable. Let $\Omega_{D}=\left\{x_{D} \mid x \in \Omega\right\}$. If $\Lambda \subset \mathbb{R}^{d}$ is a bounded Borel set, $\left(\Omega_{\Lambda}, \mathscr{F}_{\Lambda}\right)$ may be identified with $\bigcup_{\substack{n \geq 0 \\ m \geqq 0}} \Omega_{\Lambda}^{(n, m)}$,

$$
\Omega_{\Lambda}^{(n, m)} \equiv\left(\Lambda_{\mathrm{symm}}^{n} \times \Lambda_{\mathrm{symm}}^{m}\right)_{\neq},
$$


equipped with the Borel sets, where the first factor inside the parentheses refers to the $A$ particles, the second factor to the $B$ particles, $\Omega_{A}^{(0,0)}$ contains the single element $\emptyset_{\Lambda}$, the vacuum in $\Lambda$, and coincident configurations, in which two or more particles are at the same position, are not allowed. When we refer to continuous functions and to Lebesgue measure on $\Omega_{A}$ we have this identification in mind. We define a measure $d y_{\Lambda}$ on $\Omega_{\Lambda}$ by

$$
d y_{\Lambda}=\frac{1}{n ! m !} d \mathbf{y}_{1} \ldots d \mathbf{y}_{n}, d \overline{\mathbf{y}}_{1}, \ldots, d \overline{\mathbf{y}}_{m}
$$

on $\Omega_{\Lambda}^{(n, m)}$, where $y=\left(\mathbf{y}_{1}, \ldots, \mathbf{y}_{n}, \overline{\mathbf{y}}_{1}, \ldots, \overline{\mathbf{y}}_{m}\right) \in \Omega_{\Lambda}^{(n, m)}$ (and $d y_{\Lambda}$ gives unit mass to $\Omega_{\Lambda}^{(0,0)}$ ). $\Lambda$ will always refer to bounded Borel sets $\subset \mathbb{R}^{d}$.

In the Widom Rowlinson model, the only interaction is a hard core exclusion between $A$ and $B$ particles: an $A$ particle cannot be closer than a distance $R>0$ to a $B$ particle. Thus, for $F$ a Borel set $\subset \Gamma$, we let

$$
\partial F=\left\{\mathbf{r} \in \Gamma-F \inf _{\mathbf{r}^{\prime} \in F}\left|\mathbf{r}-\mathbf{r}^{\prime}\right| \leqq R\right\} .
$$

A Gibbs state for this model may be defined as a probability measure on $\Omega$ whose conditional probability given $\mathscr{F}_{\Lambda}$ is defined by the probability measure $\mu_{\Lambda}(\cdot \mid x)$ on $\Omega_{\Lambda}$ given by

$$
\begin{aligned}
& \frac{e^{-h_{\Lambda}(y, x)} d y_{\Lambda}}{\int e^{-h_{\Lambda}\left(y^{\prime}, x\right)} d y_{\Lambda}^{\prime}} \\
& h_{\Lambda}(y, x)=z^{n+m}\left(\sum_{i, k} V\left(\left|\mathbf{y}_{i}-\overline{\mathbf{y}}_{k}\right|\right)+\sum_{i, j} V\left(\left|\mathbf{y}_{i}-\overline{\mathbf{x}}_{j}\right|\right)+\sum_{k, l} V\left(\left|\overline{\mathbf{y}}_{k}-\mathbf{x}_{l}\right|\right)\right),
\end{aligned}
$$

where $y=\left(\mathbf{y}_{1}, \ldots, \mathbf{y}_{n}, \overline{\mathbf{y}}_{1}, \ldots, \overline{\mathbf{y}}_{m}\right) \in \Omega_{\Lambda}^{(n, m)}$, and $x \in \Omega, x_{\partial \Lambda}=\left(\mathbf{x}_{1}, \ldots, \mathbf{x}_{n^{\prime}}, \overline{\mathbf{x}}_{1}, \ldots, \overline{\mathbf{x}}_{m^{\prime}}\right)$, and $V(t)=\infty$ for $t<R$ and $=0$ otherwise. In particular, (1.2) is satisfied for $F=\Lambda$, and as before we say that a Gibbs $\mu$ satisfies the global Markov property if (1.2) is satisfied for all Borel sets $F \subset \Gamma$.

Note that $\mu(\cdot \mid x)$ makes sense for all functions $x \in \hat{\Omega}$, not just for $x \in \Omega$. We denote by + the function $\in \hat{\Omega}$ which everywhere has the value $+2(A$ particles everywhere) and similarly for - . The + state (or the $A$ state), the limit of the measure $\mu_{\Lambda_{n}}(\cdot \mid+)$, will be denoted by $\mu^{+}$. Similarly for the - state, $\mu^{-}$. Here, as before, $\Lambda_{n}$ denotes an increasing sequence of cubes $\subset \Gamma$ centered at the origin, with $\bigcup_{n} \Lambda_{n}=\Gamma$. We show first that the limit defining $\mu^{+}$exists, in the sense of defining a probability measure on $\Omega$. (It then follows from the argument in [9] that for sufficiently large $z$ the Gibbs states $\mu^{+}$and $\mu^{-}$are distinct.) Then we establish the global Markov property for $\mu^{+}$(and $\mu^{-}$).

The natural order on $S=\{-2,0,2\}$ induces a partial order on the set $\hat{\Omega}$ of $S$-valued functions, and in particular on $\Omega$. In this partial order $x \leqq x^{\prime}$ if $x^{\prime}$ may be obtained from $x$ by first removing some $B$ particles and then adding some $A$ particles. Similarly we have a partial order on $\Omega_{\Lambda}$. Let $\mathscr{F}_{A}^{\uparrow}\left(\mathscr{F}^{\uparrow}\right)$ denote the set of bounding increasing measurable functions on $\Omega_{\Lambda}(\Omega)$. Let $\mathscr{C}_{\Lambda}^{\uparrow} \subset \mathscr{F}_{\Lambda}^{\uparrow}$ consist of increasing functions on $\Omega_{A}$ which are either continuous or are of the form $f\left(\left\{N_{\alpha_{i}, A_{i}}\right\}_{i=1}^{n}\right), A_{i} \subset \Gamma$ a bounded set whose indicator function is Riemann integrable, $i=1, \ldots, n$. Let $\mu$ and $v$ be probability measures on $\Omega_{\Lambda}$. Then we define

$$
\mu \leqq v \Leftrightarrow \mu(f) \leqq v(f) \text { for all } f \in \mathscr{F}_{\Lambda}^{\uparrow} .
$$


The key ingredient in our results is the following:

Theorem 5.1. The Widom Rowlinson interaction is attractive in the sense that

$$
x \leqq x^{\prime} \Rightarrow \mu_{\Lambda}(\cdot \mid x) \leqq \mu_{\Lambda}\left(\cdot \mid x^{\prime}\right)
$$

for all functions $x, x^{\prime} \in \hat{\Omega}$.

Proof. It is shown in [10] by a direct computation that

$$
n_{k, l}^{\Lambda}\left(\mathbf{y}_{1}, \ldots, \mathbf{y}_{k} ; \overline{\mathbf{y}}_{1}, \ldots, \overline{\mathbf{y}}_{l} \mid x\right) \leqq z^{k+l},
$$

where the LHS is the correlation function giving the probability density of finding $k A$ particles at $\mathbf{y}_{1}, \ldots, \mathbf{y}_{k}$ and $l B$ particles at $\overline{\mathbf{y}}_{1}, \ldots, \overline{\mathbf{y}}_{l}$ for the measure $\mu_{\Lambda}(\cdot \mid x)$. It follows that

$$
\mu_{\Lambda}\left(N_{\Lambda} \geqq n \mid x\right) \leqq \frac{(z|\Lambda|)^{n}}{n !},
$$

where $|\Lambda|$ is the Lebesgue measure of $\Lambda$.

Let $\Gamma^{(\delta)}=\delta \mathbb{Z}^{d}, \delta>0$, and for $\Lambda \subset \Gamma^{(\delta)}$, let $\Omega_{\Lambda}^{(\delta)}$ be defined in the natural way. For $w \in \hat{\Omega}$, let $h_{\Lambda, w}^{(\delta)}$ be of the form (4.6) (on $\Gamma^{(\delta)}$ ) with $p=2$,

$$
\begin{array}{ll}
J(i, j)=J(i-j) & h(i)=h_{\Lambda}(i \mid w) \\
\gamma(i, j)=\gamma(i-j) & u(i)=\beta^{-1} \log z^{1 / 4} \delta^{d}+u_{\Lambda}(i \mid w)
\end{array}
$$

$i, j \in \Gamma^{(\delta)}$, in the limit defined by

$$
\begin{aligned}
& J(\mathbf{r})=\gamma(\mathbf{r})=0 \quad \text { for } \quad|\mathbf{r}| \geqq R \\
& \left.\begin{array}{l}
J(\mathbf{r})+4 \gamma(\mathbf{r})=0 \\
J(\mathbf{r})-4 \gamma(\mathbf{r})=\infty
\end{array}\right\} \quad \text { for } \quad|\mathbf{r}|<R .
\end{aligned}
$$

Here

$$
2 u_{\Lambda}(\mathbf{r} \mid w)+h_{\Lambda}(\mathbf{r} \mid w)=\left\{\begin{array}{cl}
-\infty & \text { if } \quad \mathbf{r} \in B_{w_{\mathbb{R}^{\prime \prime}}-\Lambda} \\
0 & \text { otherwise }
\end{array}\right.
$$

and

$$
2 u_{\Lambda}(\mathbf{r} \mid w)-h_{\Lambda}(\mathbf{r} \mid w)=\left\{\begin{array}{cl}
-\infty & \text { if } \mathbf{r} \in A_{\mathbb{R}^{u}-\Lambda} \\
0 & \text { otherwise }
\end{array}\right.
$$

where $A_{x}\left(B_{x}\right)$ denotes the union of open spheres of radius $R$ centered on the $A$ particles ( $B$ particles) of $x \in \hat{\Omega}$.

Let $\mu_{\Lambda, w}^{(\delta)}$ be the probability measure on $\Omega_{\Lambda}^{(\delta)}$ with density

$$
\frac{e^{-h_{\Lambda, w}^{(\delta)}(y, 0)}}{\sum_{y^{\prime} \in \Omega^{(\delta)}} e^{-h_{\Lambda}^{(\delta)}, \ldots\left(y^{\prime}, 0\right)}} .
$$

Here 0 denotes the configuration containing no particles. Let $x \in \hat{\Omega}$. As noted in [8], if $I_{A}$ is Riemann integrable, $\mu_{A}(\cdot \mid x)$ may be approximated by the lattice measures $\mu_{\Lambda^{(\delta)}, x}^{(\delta)}$, where $\Lambda^{(\delta)}=\Lambda \cap \Gamma^{(\delta)}$, in the following sense: Points $y \in \Omega_{\Lambda^{(\delta)}}^{(\delta)}$ may 
be regarded as points in $\Omega_{\Lambda}$ by extending the function $y: \Lambda^{(\delta)} \rightarrow S$ to $\Lambda$ by setting it $=0$ on $\Lambda-\Lambda^{(\delta)}$. Thus a function $f$ on $\Omega_{\Lambda}$ defines a function $f^{(\delta)}$ on $\Omega_{\Lambda^{(\delta)}}^{(\delta)}$.

Moreover, for $f \in \mathscr{F}_{\Lambda}$,

$$
\sum_{y \in \Omega^{(\delta)}} f^{(\delta)}(y) e^{-h_{A^{(\delta)}}^{(\delta)}, x_{\mathbb{R}^{d}},(y, 0)}
$$

is essentially a Riemann sum for the numerator of $\mu_{\Lambda}(f \mid x)$. Moreover, it is easy to see, by either a direct computation or the FKG inequalities, that (5.5) also holds for the lattice approximations, except $\Lambda$ on the RHS of (5.5) may have to be replaced by an appropriate bounded Borel set $\Lambda^{\prime} \supset \Lambda$ which may be chosen independent of $\delta$. It follows that for $f \in \mathscr{C}_{\Lambda}^{\uparrow}$,

$$
\mu_{\Lambda}(f \mid x)=\lim _{\delta \rightarrow 0} \mu_{\Lambda^{(\delta)}, x_{\mathbb{R}^{d}-\Lambda}^{(\delta)}}\left(f^{(\delta)}\right) .
$$

Therefore the FKG inequalities are satisfied by the measures $\mu_{\Lambda}(\cdot \mid x)$ for $f$ and $g \in \mathscr{C}_{\Lambda}^{\uparrow}$. It is shown in the Appendix that the FKG inequalities are in fact satisfied, in $\mu_{\Lambda}(\cdot \mid x)$, for all $f, g \in \mathscr{F}_{\Lambda}^{\uparrow}$, where $\Lambda$ is any bounded Borel set.

Now suppose $x \leqq x^{\prime}$. Then $x \leqq x^{\prime \prime} \leqq x^{\prime}$, where $x^{\prime \prime} \in \hat{\Omega}$ differs from $x\left(x^{\prime}\right)$ only in that some $B(A)$ particles have been removed. Let $\langle\cdot\rangle=\mu_{\Lambda}\left(\cdot \mid x^{\prime \prime}\right)$. Then

$$
\mu_{\Lambda}\left(\cdot \mid x^{\prime}\right)=\langle\cdot\rangle_{g^{\prime}}
$$

where

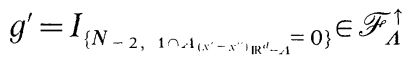

(and $x^{\prime}-x^{\prime \prime}$ is the difference of the functions $x$ and $x^{\prime}$ ). Similarly

$$
\mu_{\Lambda}(\cdot \mid x) \doteq\langle\cdot\rangle_{g},
$$

where

$$
\left.g=I_{\left\{N _ { 2 , \Lambda \cap B } \left(x-x^{\prime \prime} \mathbb{R}^{d}-A\right.\right.}=0\right\}
$$

and $-g \in \mathscr{F}_{A}^{\uparrow}$. It follows from the FKG inequalities that

$$
\mu_{\Lambda}(\cdot \mid x) \leqq \mu_{\Lambda}\left(\cdot \mid x^{\prime \prime}\right) \leqq \mu_{\Lambda}\left(\cdot \mid x^{\prime}\right)
$$

and the interaction is attractive.

That the limit (4.2) exists in the continuum now follows easily: On functions in $\bigcup_{n} \mathscr{F}_{\Lambda_{n}}^{\uparrow}$ the limit exists monotonically. Hence if the limiting values on such functions come from a probability measure on $\Omega$ at all, this measure is unique. To see that the limit comes from a probability measure, note that it follows from (5.4) that the density of $\mu_{\Lambda_{n}}(d y \mid+)$, regarded as a measure on $\Omega_{\Lambda_{m}}, m \leqq n$, w.r.t. $d y_{\Lambda_{m}}$ is $\leqq z^{k+l}$ on $\Omega_{\Lambda_{m}}^{(k, l)}$. Thus, if $d_{z} y_{\Lambda_{m}}$ is the measure on $\Omega_{\Lambda_{m}}$ given by $z^{k+l} d y_{\Lambda_{m}}$ on $\Omega_{\Lambda_{m}}^{(k, l)}$, we see that these densities are all $\in L^{\infty}\left(\Omega_{\Lambda_{m}}, d_{z} y\right)$. Therefore, by the Banach Alaoglu Theorem and a diagonalization, there exists a subsequence $n_{k}$ such that the measures $\mu_{\Lambda_{n_{k}}}(\cdot \mid+)$ converge to a probability measure on $\Omega$ on functions $\in \bigcup_{m} L^{1}\left(\Omega_{\Lambda_{m}}, d_{z} y_{A_{m}}\right)$, and in particular on functions $\in \bigcup_{m} \mathscr{F}_{\Lambda_{m}}$. (We use here the fact that probability measures on $\Omega$ are in $1-1$ correspondence with consistent families of measures on the $\Omega_{A}$ 's. This follows, for example, by regarding $\Omega$ as a 
countable product space in the natural way and applying Kolmogorov's theorem.) This proves the existence of the Gibbs state $\mu^{+}$.

That $\mu^{+}$satisfies the global Markov property may be seen by repeating, essentially verbatim, the argument in Sects. 3 and 4 . Unlike the lattice case, boundary conditions which do not correspond to configurations $x \in \Omega$ must be considered, but this makes no differences.

Theorem 5.2. The + state and the - state for the Widom-Rowlinson model satisfy the global Markov property.

Remark. The results and arguments of this section apply with minor modifications to the more general $A-B$ models described in $[10]: V_{A A}(\mathbf{r})=V_{B B}(\mathbf{r})=0, V_{A B}(\mathbf{r}) \geqq 0$ (where $V_{A A}, V_{B B}$, and $V_{A B}$ are respectively the pair potential between two $A$ particles, two $B$ particles, and an $A$ particle and a $B$ particle), with external fields for the $A$ and $B$ particles which are bounded below [reflected in bounded activities $z_{A}(\mathbf{r})$ and $\left.z_{B}(\mathbf{r})\right]$. The main differences are the following:

(a) The RHS of (5.4) should be replaced by $z_{A}\left(\mathbf{y}_{1}\right) \ldots z_{A}\left(\mathbf{y}_{k}\right) z_{B}\left(\overline{\mathbf{y}}_{1}\right) \ldots z_{B}\left(\overline{\mathbf{y}}_{l}\right)$.

(b) The lattice approximation should be carried through for continuous potentials and fields and the results extended to Borel potentials and fields by a density argument.

(c) $\mu_{\Lambda}(\cdot \mid x)$ will now make sense for Borel measurable $x: \mathbb{R}^{d} \rightarrow S$, but not necessarily for all $x: \mathbb{R}^{d} \rightarrow S$. Theorem 5.1 will hold for Borel measurable $x$ and $x^{\prime}$.

\section{Appendix}

The following result is proven in [11].

Theorem A1. Let $T$ be a finite partially ordered set, and let $\mu_{1}$ and $\mu_{2}$ be probability measures on $T$. Then the following are equivalent:

(i) $\mu_{1}(f) \leqq \mu_{2}(f)$ for all increasing functions $f: T \rightarrow \mathbb{R}$.

(ii) There exists a probability measure $v$ on $T \times T$ such that

(a) $v(\cdot \times T)=\mu_{1}$,

(b) $v(T \times \cdot)=\mu_{2}$,

and

(c) $v\{(x, y) \in T \times T \mid x \leqq y\}=1$.

We wish to extend this result to infinite partially ordered sets. What we can prove is the following:

Theorem A2. Let $(B, \mathscr{B})$ be a partially ordered (standard) Borel space $[12,13]$. Let $f_{n}$ be a sequence of increasing, $\mathscr{B}$-measurable $\{0,1\}$ valued functions on $B$ which (1) separates points and (2) determines the partial order [i.e., $x \leqq y \Leftrightarrow f_{n}(x) \leqq f_{n}(y)$ for all $n]$. Let $\mathscr{B}^{\uparrow}$ be the set of bounded, increasing $\mathscr{B}$-measurable functions and let $\hat{\mathscr{B}}^{\dagger}$ be the set of increasing functions of the form $\psi\left(f_{1}, \ldots, f_{n}\right)$ for some $n$. Let $\mu_{1}$ and $\mu_{2}$ be 
probability measures on $B$. Then the following are equivalent:

(i) $\mu_{1}(f) \leqq \mu_{2}(f)$ for all $f \in \mathscr{B}^{\dagger}$.

(ii) $\mu_{1}(f) \leqq \mu_{2}(f)$ for all $f \in \hat{\mathscr{B}}^{\dagger}$.

(iii) There exists a probability measure $v$ on $B \times B$ such that

(a) $v(\cdot \times B)=\mu_{1}$,

(b) $v(B \times \cdot)=\mu_{2}$,

and

(c) $v\{(x, y) \in B \times B \mid x \leqq y\}=1$.

Proof. (i) $\rightarrow$ (ii) is trivial. To see that (ii) $\rightarrow$ (iii) consider the function $\mathbf{f}: B \rightarrow\{0,1\}^{\infty}$, $\mathbf{f}(x)=\left(f_{1}(x), f_{2}(x), \ldots\right), x \in B$. $\mathbf{f}$ is a measurable injection from the (standard) Borel space $(B, \mathscr{B})$ to the standard Borel space $\{0,1\}^{\infty}$ with the product $\sigma$-algebra. Therefore $\mathbf{f}$ takes Borel sets into Borel sets [13], and in particular $\mathbf{f}(B) \equiv \hat{B}$ is Borel. f is thus an isomorphism $(B, \mathscr{B}, \leqq) \rightarrow(\hat{B}, \leqq)$ and we may thus assume without loss of generality, that $B$ is a Borel subset of $\{0,1\}^{\infty}$ and that the functions $f_{n}$ are the coordinates. Let $B_{n}=\{0,1\}^{n}, n=1,2, \ldots$, be the set of values of the first $n$ coordinates. Then by Theorem A1, there exists a probability $v_{n}$ on $B_{n}$ satisfying

(a) $v_{n}\left(\cdot \times B_{n}\right)=\mu_{1} \quad$ on $B_{n}$,

(b) $v_{n}\left(B_{n} \times \cdot\right)=\mu_{2}$ on $B_{n}$,

and

(c) $\quad v_{n}\left\{(x, y) \in B_{n} \times B_{n} \mid x \leqq y\right\}=1$.

There exists a subsequence $n_{j}$ such that $v_{n_{j}}$ converges weakly to a probability measure $v$ on $B \times B$. It is easy to see that $v$ must satisfy (a)-(c) of the theorem.

(iii) $\rightarrow$ (i) because for $f \in \mathscr{B}^{\dagger}$

$\mu_{1}(f)=\int v(d x, d y) f(x) \leqq \int v(d x, d y) f(y)=\mu_{2}(f)$.

$(\Omega, \mathscr{F})$ and $\left(\Omega_{A}, \mathscr{F}_{A}\right)$ are standard Borel spaces. This follows, for example, from the product structure of these spaces and the stability properties of standard Borel spaces [13], or from the fact that $\mathscr{F}\left(\mathscr{F}_{A}\right)$ is the set of Borel sets for a Polish space topology on $\Omega\left(\Omega_{A}\right)[14,13]$. Suppose $(B, \mathscr{B})=\left(\Omega_{A}, \mathscr{F}_{A}\right), \Lambda$ a bounded Borel set. It is easy to find a sequence of $\{0,1\}$-valued functions $\in \mathscr{C}_{A}^{\uparrow}$ satisfying (1) and (2) of Theorem A2. (For example, the collection of functions which are either of the form $I_{\left\{N_{2, D}>0\right\}}$ or of the form $I_{\left\{N_{-2, D}=0\right\}}$, where $D$ ranges over open spheres of rational radius whose center has rational coordinates, will do.) Let $\langle\cdot\rangle=\mu_{\Lambda}(\cdot \mid x)$ for the Widom-Rowlinson model, and let $g \in \mathscr{C}_{\Lambda}^{\uparrow}, g \geqq 0,\langle g\rangle \neq 0$. Suppose first that $\Lambda$, i.e., $I_{A}$, is Riemann integrable. It was shown in Sect. 5 that the FKG inequalities are satisfied for functions $\in \mathscr{C}_{A}^{\dagger}$, and therefore (ii) of Theorem A2 is satisfied by $\mu_{1}=\langle\cdot\rangle$ and $\mu_{2}=\langle\cdot\rangle_{g}$. It follows by an application of the theorem that the FKG inequalities are satisfied for all functions $f \in \mathscr{F}_{A}^{\dagger}, g \in \mathscr{C}_{\Lambda}^{\dagger}$, and by another, similar, application of the theorem, for all functions $f, g \in \mathscr{F}_{A}^{\dagger}$. Finally, any bounded Borel set may be approximated by Riemann integrable sets $\Lambda^{\prime}$. It follows, using (5.5) for $n=1$, that $\mu_{\Lambda}(\cdot \mid x)$ is approximated by $\mu_{\Lambda^{\prime}}\left(\cdot \mid x^{\prime}\right)$ for appropriately chosen $x^{\prime}$. Therefore, the restriction that $\Lambda$ be Riemann integrable can be removed. 
Theorem A3. In the Widom-Rowlinson model

$$
\langle f g\rangle \geqq\langle f\rangle\langle g\rangle \text {, }
$$

where $\langle\cdot\rangle=\mu_{\Lambda}(\cdot \mid x), x \in \hat{\Omega}, \Lambda$ a bounded Borel set, and $f, g \in \mathscr{F}_{\Lambda}^{\uparrow}$.

Acknowledgements. The author wishes to thank Jean Bricmont and Joel Lebowitz for useful discussions and suggestions.

\section{References}

1. Dobrushin, R.L.: Funct. Anal. Appl. 2, 291 (1968)

2. Lanford, O.E., III, Ruelle, D. : Comm. Math. Phys. 13, 194 (1969)

3. Albeverio, S., Høegh-Krohn, R. : The global Markov property for lattice systems. (Submitted to Z. Wahr. Verw. Geb.)

4. Föllmer, H. : On the global Markov property. Preprint 1978

5. Dobrushin, R.L.: Theory Prob. Appl. 17, 582 (1972)

6. Fortuin, C.M., Kasteleyn, P.W., Ginibre, J.: Commun. Math. Phys. 22, 89 (1971)

7. Battle, G.A., Rosen, L.: J. Stat. Phys. 22, 123 (1980)

8. Lebowitz, J.L., Monroe, J.L. : Commun. Math. Phys. 28, 301 (1972)

9. Ruelle, D.: Phys. Rev. Lett. 27, 1040 (1971)

10. Cassandro, M., Gallavotti, G., Lebowitz, J.L., Monroe, J.L.: Commun. Math. Phys. 32, 153 (1973)

11. Preston, C.J.: Commun. Math. Phys. 36, 233 (1974)

12. Breiman, L.: Probability. London: Addison-Wesley 1968

13. Schwartz, L.: Radon measures. London, New York: Oxford University Press 1973

14. Lanford, O.E., III.: Commun. Math. Phys. 11, 257 (1969)

Communicated by E. Lieb

Received December 18, 1979 